\title{
FREQUENCY DRIFT AND FINE STRUCTURE OF 200-MC/S SOLAR BURSTS
}

\author{
Ø. ELGARØY \\ Institute of Theoretical Astrophysics, Oslo, Norway
}

In 1957 high-speed records were obtained at a frequency of $200 \mathrm{Mc} / \mathrm{s}$ during solar radio noise storms at the Solar Observatory at Harestua. For the registrations a receiver of bandwidth $0.3 \mathrm{Mc} / \mathrm{s}$ and time constant 0.01 seconds was used in connection with a Brush recorder. Most records were secured at a paper speed of $25 \mathrm{~mm} / \mathrm{second}$.

1. DURATION OF PIPS

Storm bursts, recorded with an ordinary low-speed recorder, are resolved into several pips of short durations on high-speed records. Because of the complex structure of a noise storm, duration measurements of these pips are

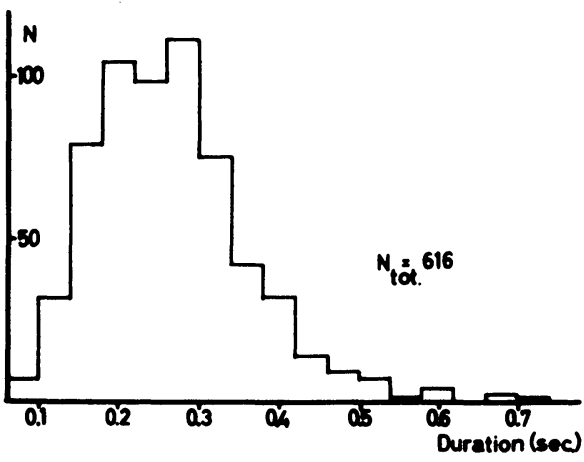

Fig. 1. The distribution of pips of different half-widths. somewhat complicated. Individual pips overlap to a great extent; therefore to determine the duration of single pips, we had to select those least interfered with by neighboring ones. The duration was measured between half-power points. On nine days in the period 1957 April to August the lengths of the pips were determined and the mean duration calculated. Slight variations were present in the mean values for different days. The mean duration of the total number of pips, about 600 , was $t=(0.266 \pm 0.004)$ seconds. The distribution of pips of different half-widths is given in Fig. 1. The histogram shows a peak around a duration of 0.25 seconds and durations of more than say 0.5 seconds are rare.

\section{DISTANCE BETWEEN PIPS}

The pips show a tendency to cluster into groups, in which the distance between successive pips has been measured; a mean value of $T=(0.46 \pm 0.01)$ seconds is found in material consisting of nearly 900 pips. For 250 pips the duration versus time-interval to next peak was plotted. The diagram indicated a linear relation; an application of the method of least squares gave the result $T=2 t$; i.e., pips of long durations are followed by a longer interval than short pips. This is typical of saturation phenomena. 


\section{FREQUENCY DRIFT}

More information about the pips could be gained by simultaneous registrations on two appropriately spaced frequencies. An experimental version of a double-channel receiver, therefore, was put into operation and run in selected periods. The channels were centered at 199 and $200.5 \mathrm{Mc} / \mathrm{s}$, respectively, and the bandwidth of each was adjusted to $0.3 \mathrm{Mc} / \mathrm{s}$. Records were taken at paper speeds of 5,25 , and $125 \mathrm{~mm} /$ second and several thousand feet of recording paper were run.

Reduction of the recordings brought out that most pips occurred on the two frequencies at slightly different times. The pips could occur first on the high frequency, on the low frequency, or simultaneously. The term "time of occurrence" signifies in this connection the time of a pip's maximum intensity. In all, a little more than 2000 pairs of pips have been analysed. In this material 48 per cent of the pips occurred first on the low frequency, 34 per cent occurred first on the high frequency, and 18 per cent occurred simultaneously. When the same active center was followed for two or more days, the number of pairs of pips occurring first on the high frequency clearly decayed with time, whereas those pairs of pips appearing first on the low frequency occurred more frequently. This is shown in Fig. 2 for the days June 3 and 4 . If we tentatively assume a linear variation with time of the percentages of pips occurring first on the high frequency and of those occurring first on the low frequency, the intersection point of the lines combining corresponding numbers on the two days falls quite near to the time of central meridian passage of the believed storm-producing area. Within limits of uncertainty this seems to be true in four more cases. Only observations on April 28 and 30 disagree, but it should be mentioned that on these days probably different noise sources have been observed. Hence, during central meridian passage of an active radio noise area, at least in some cases, equal amounts of pips should be found occurring first on the high and the low frequency. Further measurement on this point is desirable. The effect of new active areas on the distribution of pairs of pips starting on the higher or the lower frequency is illustrated in Fig. 3. From April 4 to 8 the same noise source is followed, but between April 8 and 9 an abrupt change takes place which coincides with a development of new centers of activity.

The time-differences between corresponding pips on the two neighboring frequencies $199 \mathrm{Mc} / \mathrm{s}$ and $200.5 \mathrm{Mc} / \mathrm{s}$ may be interpreted as being due to a frequency drift. This suggestion is

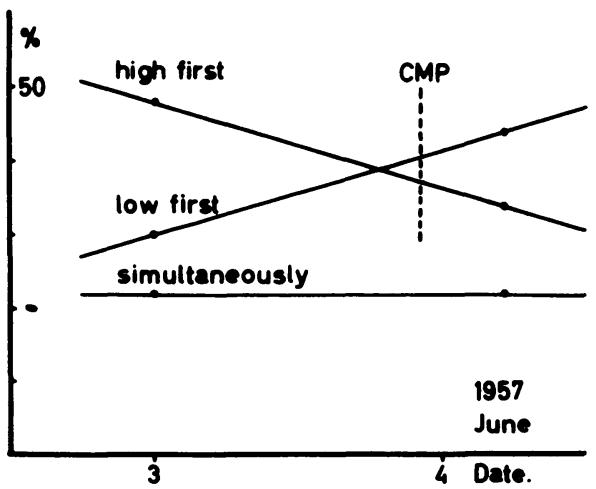

FIG. 2. Plot of observed percentages of pips occurring first on the high frequency, the low frequency, or simultaneously versus time for the days June 3 and 4. 


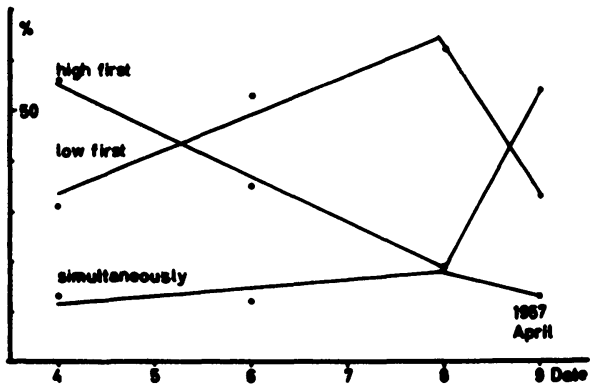

FIG. 3. Change in the distribution of pips occurring first in the high- and the low-frequency channel when new active areas develop and contribute to the radiation.

supported by some preliminary records which were taken recently with a narrow-band swept receiver at Harestua during a solar noise storm. Figs. 4 and 5 both clearly show that short-lived bursts of small bandwidth may drift from higher to lower frequencies or vice versa.

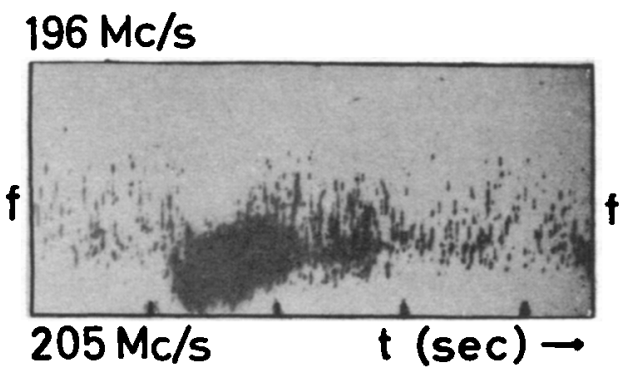

FIG. 5. Burst with frequency drift from lower to higher frequencies.

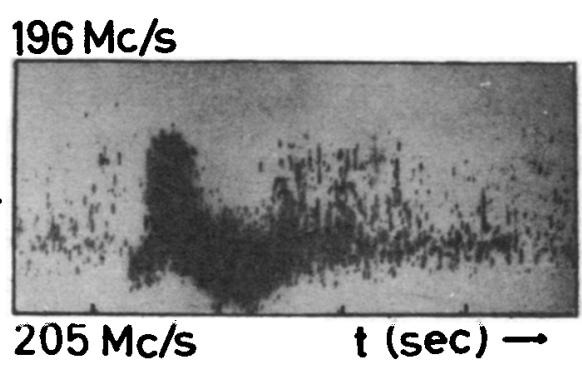

FIG. 4. Burst showing frequency drift from higher to lower frequencies.

\section{Discussion}

Minnaert: The paper of Mr. Elgarøy and that of de Groot are closely similar and nicely complement each other, the one having been made at 400 , the other at $200 \mathrm{Mc} / \mathrm{s}$. Is there any explanation for the curious phenomena described by Mr. Elgarøy (before central meridian, high-frequency first; after meridian passage, the other way round)?

Elgarøy: I have no explanation for this fact.

de Jager: The mean errors given by you for the time interval between two successive pips was not confirmed by our measurements, which show that great time differences may occur between two pips. They certainly do not follow each other in time intervals of exactly 0.46 seconds. A similar remark applies to the duration of the pips. The histogram of pip duration can change from day to day, suggesting that at $200 \mathrm{Mc} / \mathrm{s}$ pips of different types occur, with frequency maxima in the duration histograms as high as 1.0 seconds.

Elgarøy: The errors given are root-mean-square errors. Mean durations might vary, but not very much on different days. The distance between successive pips in groups may vary to a higher degree. 